\title{
Intravesical electro-osmotic administration of mitomycin C
}

\author{
Savino M. Di Stasi ${ }^{1}$, Cristian Verri ${ }^{2}$, Francesco Celestino ${ }^{2}$, Francesco De Carlo ${ }^{1}$, Vincenzo Pagliarulo ${ }^{3}$ \\ ${ }^{1}$ Department of Surgery and Experimental Medicine, Tor Vergata University, Rome - Italy \\ ${ }^{2}$ Operative Unit of Urologic Oncology, Policlinico Casilino, Rome - Italy \\ ${ }^{3}$ Department of Emergency and Organ Transplantation, University of Bari, Bari - Italy
}

\begin{abstract}
Bladder cancer is very common and most cases are diagnosed as nonmuscle invasive disease, which is characterized by its propensity to recur and progress. Intravesical therapy is used to delay recurrence and progression, while cystectomy is reserved for patients who are refractory to transurethral resection and intravesical therapy. There is an increasing interest in methods to enhance the delivery of intravesical chemotherapeutic agents to improve efficacy. In vitro and in vivo studies demonstrated that electro-osmosis of mitomycin C (MMC) is more effective in delivering this drug into the urothelium, lamina propria, and superficial muscle layers of the bladder wall than is passive transport. Higher MMC tissue concentrations might have a clinical impact in the treatment of nonmuscle invasive bladder cancer (NMIBC). In randomized trials, intravesical electro-osmotic MMC was associated with superior response rate in high-risk NMIBC cancer, compared with passive diffusion MMC transport. New strategies such as intravesical Bacillus Calmette-Guerin (BCG) combined with electro-osmotic MMC as well as intravesical pre-operative electro-osmotic MMC provided promising results in terms of higher remission rates and longer remission times.

Device-assisted intravesical chemotherapy may be a useful ancillary procedure in the treatment of NMIBC. Its evaluation must be planned with respect to the technical functioning of equipment and their use for a clear purpose to avoid the financial and human costs associated with incorrect therapies.
\end{abstract}

Keywords: Bladder cancer, Device-assisted intravesical chemotherapy, Intravesical electro-osmotic administration, Mitomycin C, Nonmuscle invasive bladder cancer

\section{Introduction}

Bladder cancer is the seventh most common cancer in men (1). In 2012, an estimated 429,793 cases were diagnosed and 165,068 patients died of the disease worldwide (1). When initially diagnosed, most bladder cancers are non muscle invasive, including papillary lesions confined to the urothelium (stage Ta) or invading the lamina propria (stage T1), and carcinoma in situ (stage Tis) (2). Transurethral resection of the bladder tumors (TURBT) is the standard initial diagnostic and therapeutic step. However, around 53\% of patients undergoing TURBT alone recur within 2 years (3). Intravesical therapy is used in an attempt to improve the

Accepted: September 13, 2016

Published online: October 1, 2016

Corresponding author:

Professor Savino M. Di Stasi

Department of Surgery and Experimental Medicine

Tor Vergata University

00173 Rome, Italy

sdistas@tin.it recurrence-free interval (2). Such therapy can take the form of either chemotherapy with a cytotoxic agent or immunotherapy with bacillus Calmette-Guerin (BCG). It can be administered as a single, "peri-operative" dose at the time of TURBT or else as an "adjuvant" dosing over a period of time after TURBT. Despite adjuvant intravesical therapy, $45 \%$ of patients will have tumor recurrence within 12 months and $3-15 \%$ progress to muscle invasive disease within 5 years (2). Moreover, repeating TURBT and intravesical instillations of immunotherapeutic and chemotherapeutic drugs cause substantial inconvenience and morbidity, making cost per patient from diagnosis to death the highest of all cancers (4). Therefore, the improvement of effectiveness and cost-efficacy of adjuvant intravesical therapy remains a significant medical need.

Mitomycin C (MMC) has become the most commonly used intravesical cytotoxic agent and is the most familiar to urologists in routine practice. Despite its relative popularity in the treatment of patients with NMIBC, many questions remain about the optimal use of MMC. Although many aspects of the MMC instillation regimen are empirical and lack robust data to support their use, research into some of these limiting factors has revealed means to optimize MMC delivery (5). Strategies to enhance delivery of MMC therapy have been studied 
in detail, and different approaches, including electro-osmotic administration (6), have been recommended for implementation in routine clinical practice (7).

\section{Principles of intravesical electro-osmotic administra- tion of mitomycin C}

The principle of intravesical administration of MMC with an electric current is a combination of several different electromolecular interactions: iontophoresis, electro-osmosis, and electroporation are all responsible for electromotive transport of drug molecules in an electric field across biological membranes into underlying tissues (8). lontophoresis refers to accelerated ion transport into tissues by means of an electric current passed through a solution containing the ions to be administered at a rate defined by Faraday's Law (9). Usually, iontophoresis is associated with increased transport of water that will entrain any non-ionized solutes present, a phenomenon termed electro-osmosis. Electro-osmosis is used to describe the current-induced convective flow of water in association with ions, which can accelerate the transport of ionized molecules down coulombic gradients, non-ionized polar molecules (10), and ionized molecules against their coulombic gradients (11). Electroporation implies increasing biological membrane permeability under the influence of an electric field, which increases transport rates down concentration gradients (12). Drug transport rate is the algebraic sum of that induced by passive diffusion and by electromotive transport, but, when dealing with a membrane of low permeability such as the urothelium, electroosmotic drug administration is so dominant that it may be considered as the sole force manipulating drug transport. The administration rates are markedly increased and they are controllable by varying the current intensity. $\mathrm{MMC}$ is almost non-ionized within the full $\mathrm{pH}$ range tolerated by the bladder: 4.5-8.5 units (13), but some preparations contain $\mathrm{NaCl}$ as an excipient. When these vials are dissolved in water, the $\mathrm{NaCl}$ conducts current, and the polar, non-ionized MMC is administered by electro-osmosis. Therefore, realistic clinical or laboratory investigations involve mainly the electro-osmosis (14) and probably electroporation (12). The requisite ions for this mode of drug administration come as a sodium chloride excipient in the MMC crystals supplied. When current of positive polarity is applied to a solution containing $\mathrm{Na}^{+} / \mathrm{Cl} /$ $M M C$, sodium ions are iontophoresed into underlying tissues, a process that includes transport of water in the form of hydration shells around $\mathrm{Na}^{+}(15)$ as well as in the free form (9). Solubilized, non-ionized MMC is entrained in this water flux in quantities proportional to its concentration in solution.

MMC penetration of the bladder wall has been investigated in laboratory in a two-cell diffusion chamber model (16). Tissue sections of human bladder were inserted into two chamber cells with urothelium exposed to donor compartments containing MMC $(10 \mathrm{mg}$ in $100 \mathrm{ml}$ of $0.24 \% \mathrm{NaCl}$ solution) and an anode and with serosa exposed to receptor compartments containing $100 \mathrm{ml}$ of $0.9 \% \mathrm{NaCl}$ solution and a cathode (17). Fourteen paired experiments (current $5 \mathrm{~mA} /$ no current) were conducted over $15 \mathrm{~mm}$. MMC concentrations in human bladder tissue sections were analyzed by high-performance liquid chromatography. Tissue viability and morphology and MMC stability were assessed by trypan blue exclusion test, histological analysis, and mass spectrometry analysis.

This initial study showed that in all tissue samples exposed to electric current, MMC concentrations were higher than after passive diffusion (PD) $(21.5 \pm 8.4 \mathrm{vs} .3 .4 \pm 1.9 \mu \mathrm{g} / \mathrm{g}$, $p=0.0010$ ), the variability in drug delivery rate was reduced ( $39.1 \%$ vs. $56.7 \%, p=0.0010$ ), and the electric current caused no histological tissue damage and no chemical modification to MMC.

In a second study, MMC concentration-depth profiles in the bladder wall after passive diffusion and electro-osmotic administration were compared (14). During each paired experiment, two tissue samples from a single patient were placed between the two chambers of individual diffusion cells.

MMC (80 mg) and $1920 \mathrm{mg}$ of $\mathrm{NaCl}$ were dissolved in $200 \mathrm{ml}$ of bidistilled $\mathrm{H}_{2} \mathrm{O}$, and then the solution was divided into two volumes of $100 \mathrm{ml}$ each $(40 \mathrm{mg}$ of $\mathrm{MMC}$ in $\mathrm{NaCl}$ $0.96 \%$ solution), which were placed in paired passive diffusion and electro-osmotic administration donor compartments. The receptor compartments were filled with $100 \mathrm{ml} \mathrm{NaCl}$ solution $0.9 \%$. In electro-osmotic administration experiments, an anode was placed in the donor compartment and a cathode in the receptor compartment. The electrodes were connected to the current generator and experiments were performed with pulsed direct current of $20 \mathrm{~mA}$ for a duration of 30 minutes. No electric current was applied in passive diffusion control experiments. Each tissue segment was rapidly weighed and frozen by placement of the urothelial side on a flat stainless-steel plate cooled on liquid nitrogen. The frozen tissue was glued onto the cryotome object holder and the urothelial surface was exposed for sectioning. The first $80 \mu \mathrm{m}$ of tissue directly in contact with the MMC solution was trimmed off to avoid contamination by the dosing solution. Tissue was sectioned into $40 \mu \mathrm{m}$ thicknesses, placed, and stored frozen. For tissues between 80 and $200 \mu \mathrm{m}$ in depth (urothelium), each $40-\mu \mathrm{m}$ segment was analyzed individually. For tissue between 200 and $1200 \mu \mathrm{m}$ in depth (lamina propria) and beyond $1200 \mu \mathrm{m}$ in depth (muscularis), five and ten segments, respectively, were pooled for analysis. Bladder wall sections were analyzed by high-performance liquid chromatography for MMC concentration. Tissue viability and morphology and MMC stability were assessed by trypan-blue exclusion test, histological examination, and mass spectrometry analysis. MMC concentrations reached a plateau in all compartments after 15 mintes with no further increase during the 60 minutes of incubation. The mean concentration of MMC transported into the bladder wall by electro-osmotic administration $(28.2 \pm 4.0 \mu \mathrm{g} / \mathrm{g})$ significantly exceeded the concentration achieved by passive diffusion $(5.3 \pm 0.8 \mathrm{mg} / \mathrm{g})$, which is mirrored by the respective concentrations observed in the urothelium (170.0 \pm 19.6 vs. $46.6 \pm 8.6 \mu \mathrm{g} / \mathrm{g}$ ), lamina propria ( $65.6 \pm 5.1 \mathrm{vs} .16 .1 \pm 2.6 \mu \mathrm{g} / \mathrm{g}$ ), and superficial muscle layers ( $15.9 \pm 3.5$ vs. $1.9 \pm 0.4 \mu \mathrm{g} / \mathrm{g}$ ). The variability of MMC delivery was markedly reduced with electro-osmotic administration as compared with passive diffusione in all except the superficial muscle layer. It was concluded that electro-osmotic administration significantly enhanced $\mathrm{MMC}$ administration and reduced variability in drug delivery rate in all bladder wall layers, reaching IC90 levels (18) in the urothelium and lamina propria. 
Much more convincing are in vivo data from a clinical study that compared intravesical MMC administered by passive diffusion or electro-osmotic administration in high-risk NMIBC (19). A total of 15 patients in the intravesical passive diffusion and electro-osmotic MMC arms, respectively, underwent pharmacological evaluation during the first intravesical instillation. Blood samples of $10 \mathrm{ml}$ were drawn before and at certain time points after MMC instillation, including $0,5,15$, $30,45,60,90,120,180,300$, and 480 minutes. In both arms, passive and electro-osmotic MMC, bladder content samples were obtained at $5,15,30,45$, and 60 , and 5, 15, and 30 minutes, respectively. Peak plasma MMC concentrations were significantly higher with electro-osmotic administration than passive diffusion ( $42.9 \pm 7.1$ vs. $7.8 \pm 1.7 \mathrm{ng} / \mathrm{ml})$. These data were consistent with bladder content absorption: for passive diffusion, the absorption process was almost complete within 15 minutes, and at 60 minutes, approximately $50 \%$ of the initial MMC content was absorbed; with electro-osmotic administration at 30 minutes, more than $80 \%$ of the initial MMC content was absorbed. Although intravesical electro-osmotic administration markedly increased drug transport into the bladder wall, MMC plasma levels remained well below toxic concentrations (400 $\mathrm{ng} / \mathrm{ml})$.

\section{Efficacy of intravesical electro-osmotic MMC}

In a phase II study by Brausi et al (20), 28 patients with multifocal stage Ta-T1 and grade 1-2 bladder tumors underwent TURBT, except for one lesion left as a marker lesion. Following TURBT, 13 patients received intravesical passive diffusion MMC instillation $(40 \mathrm{mg}$ in $50 \mathrm{ml}$ of water for 120 minutes), once a week for 8 weeks, and 15 patients intravesical electro-osmotic MMC (40 mg in $50 \mathrm{ml}$ of water and $15 \mathrm{~mA}$ electric current for 30 minutes) once a week for 8 weeks. The marker lesion disappeared in five of 12 patients (41.6\%) after passive diffusion MMC and in six of 15 patients (40\%) after electro-osmotic MMC. Recurrence rate and disease-free interval were $60 \%$ and 10.5 month in passive diffusion MMC group and 33\% and 14.5 months in the electro-osmotic MMC group, respectively.

Another observational study on patients with NMIBC reported low recurrence rates after intravesical electro-osmotic MMC (40 mg in $50 \mathrm{ml}$ water with $15 \mathrm{~mA}$ for 20 minutes) given once a week for 4 weeks (21). After a median follow-up of 14.1 months, nine of 16 patients (56\%) were recurrence free.

In aprospective non-randomized study, the efficacy of intravesical MMC administered by passive diffusion $(40 \mathrm{mg}$ in $50 \mathrm{ml}$ saline for 60 minutes), or electro-osmotic administration $(40 \mathrm{mg}$ in $150 \mathrm{ml}$ distilled water with $20 \mathrm{~mA}$ for 20 minutes) or in combination with local microwave-induced hyperthermia (40 $\mathrm{mg}$ in $50 \mathrm{ml}$ distilled water delivered at a mean temperature of $42.5^{\circ} \mathrm{C}$ for 60 minutes) was compared (22). Eighty patients with single, recurrent, low-stage, lowgrade, and stage Ta-T1 tumors were enrolled. In all patients, the treatment was scheduled as a neo-adjuvant regimen prior to TURBT, to evaluate the ablative potential against a marker lesion, and included four weekly sessions. Complete response was observed in $27.7 \%$ (10/36 patients) after MMC alone vs. $40.0 \%(6 / 15)$ after electro-osmotic administration and $66.0 \%$ $(19 / 29)$ after local microwave-induced hyperthermia. Side effects were more prominent in the local microwave-induced hyperthermia arm than in the electro-osmotic and passive diffusion arms. It should be emphasized that patients were free to choose their treatment and were not followed for long-term risk of recurrence or progression.

Despite short follow-up times and small patient cohorts, findings in these studies suggested that electro-osmotic MMC was associated with better clinical outcomes than MMC passive diffusion instillation protocols.

In a prospective randomized study, response rates for intravesical passive diffusion MMC $(40 \mathrm{mg} / 100 \mathrm{ml}$ water for 60 minutes), electro-osmotic MMC ( $40 \mathrm{mg} / 100 \mathrm{ml}$ water with $20 \mathrm{~mA}$ electric current for 30 minutes), and BCG $(81 \mathrm{mg} / 50 \mathrm{ml}$ saline fo 120 minutes) were compared in 108 patients with multifocal Tis (19). All patients received a 6-week intravesical treatment course. Non-responders received another 6-week course of therapy, while responders received 10 monthly maintenance treatments. The results of this study suggested that, in terms of complete response and disease-free interval, intravesical electro-osmotic MMC and BCG were effective treatments for carcinoma in situ of the bladder (Tab. I). Both seemed more efficacious than passive MMC transport. However, progression to muscle invasive disease, time to progression, and overall as well disease-specific mortality were not significantly different between the three arms. As the response rates overlapped, electro-osmotic $M M C$ may be an alternative or rather a complementary therapy, to BCG. Peak plasma MMC was significantly higher after electro-osmotic MMC than passive diffusion MMC, indicating bladder content absorption. Side effects were significantly more prominent in the BCG arm and there were no statistical differences between the two MMC arms.

\section{Promising new paradigms to improve the efficacy of intravesical MMC}

As a consequence of the results of the previous study (19), a randomized trial comparing intravesical BCG alone with sequential BCG/electro-osmotic MMC in patients with pT1 bladder cancer was conducted (23). It was hypothesized that the use of BCG before MMC would induce inflammation and enhance tissue uptake of MMC. MMC instillations were based on their potential to eliminate malignant cells that escaped the immunological surveillance of BCG. After TURBT and multiple biopsies, 212 patients with stage pT1 bladder cancer, $39 \%$ of whom had grade 3 disease, were randomly assigned to $81 \mathrm{mg} \mathrm{BCG}$, suspended in $50 \mathrm{ml}$ saline solution and infused over 120 minutes once a week for 6 weeks ( $n=105)$; or to $81 \mathrm{mg} \mathrm{BCG}$ infused over 120 minutes once a week for 2 weeks, followed by $40 \mathrm{mg}$, dissolved in $100 \mathrm{ml}$ water, electro-osmotic MMC (intravesical electric current $20 \mathrm{~mA}$ for 30 minutes) once a week as one cycle for three cycles ( $\mathrm{n}$ $=107$ ). Complete responders underwent maintenance treatment: those assigned BCG alone had one infusion of $81 \mathrm{mg}$ BCG once a month for 10 months, and those assigned sequential BCG and MMC had $40 \mathrm{mg}$ electro-osmotic MMC once a month for 2 months, followed by $81 \mathrm{mg} \mathrm{BCG}$ once a month as one cycle for three cycles (Fig. 1). Median followup was 88 months. Patients assigned to sequential BCG/ electro-osmotic MMC had a significantly higher disease-free 
TABLE I - Comparison of the outcomes of the study endpoints from the randomized trial comparing intravesical passive diffusion MMC, electro-osmotic MMC, and BCG in patients with multifocal carcinoma in situ of the bladder (19)

\begin{tabular}{|c|c|c|c|c|}
\hline & $\mathrm{PD} / \mathrm{MMC}$ & EMDA/MMC & BCG & $\mathbf{p}$ \\
\hline \multicolumn{5}{|l|}{ Complete response (\%) } \\
\hline - 3 months & $10 / 36(27.8)$ & $19 / 36(52.8)$ & $20 / 36(55.5)$ & 0.036 \\
\hline - Crossover & - & $3 / 13(23.1)$ & $14 / 40(35.0)$ & 0.511 \\
\hline Median follow-up, months & 84 & 94 & 79 & 0.94 \\
\hline - No. of patients (\%) & $30 / 36(83.3)$ & $24 / 36(66.7)$ & $24 / 36(66.7)$ & 0.221 \\
\hline - Median time to recurrence, months & 15.1 & 44.8 & 33.3 & $<0.0001$ \\
\hline \multicolumn{5}{|l|}{ Disease progression } \\
\hline - No. of patients (\%) & $16 / 36(44.4)$ & $11 / 36(30.6)$ & $10 / 36(27.8)$ & 0.302 \\
\hline - Median time to progression, months & 27.5 & 68.8 & 70.0 & 0.064 \\
\hline
\end{tabular}

\section{Intravesical BCG Alone}

$\nabla$ BCG

$\triangle \mathrm{MMC}$
Fig. 1 - Dose schedules of a randomized study comparing intravesical BCG alone with sequential BCG/electro-osmotic mitomycin $\mathrm{C}$ (MMC) in patients with pT1 bladder cancer (23).

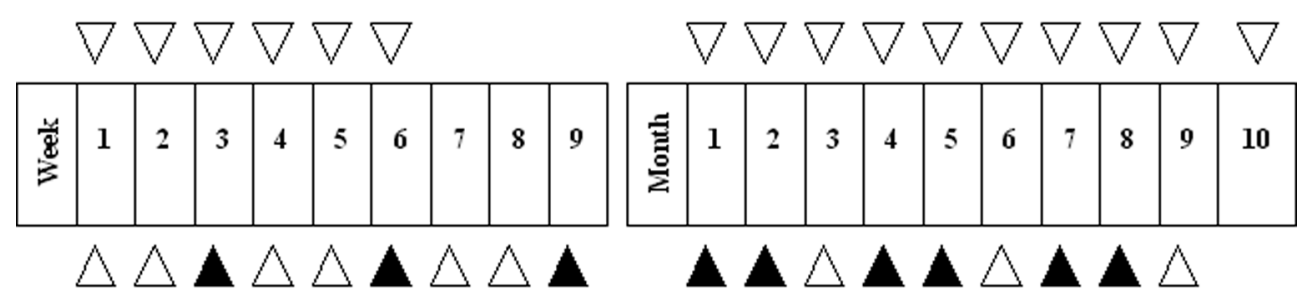

\section{Intravesical sequential BCG/MMC}

interval than patients assigned to BCG alone (Tab. II). Patients assigned to sequential BCG/electro-osmotic MMC also had significantly lower rates of recurrence, disease progression, overall mortality, and disease-specific mortality (Tab. II). There were no significant differences in the toxic side effects in the two groups. It was concluded that BCG-induced inflammation might increase urothelial permeability so that MMC was able to reach its target tissue more easily and exert its anticancer effect more efficaciously.

Recently, Gan et al (24) conducted a prospective, nonrandomized trial aimed to determine the efficacy and tolerability of sequential BCG/electro-osmotic MMC, as the standard induction regime in 107 patients with high-risk NMIBC. The full induction schedule was not completed in 30 of 107 patients (28\%). A complete response rate of $87 \%$ was reported, with $86 \%$ and $93 \%$ of patients remaining disease free at 1 and 2 years, respectively. There was no significant difference in recurrence rates between patients who completed the full schedule or had a reduced schedule at first check, 1-year, or 2-year cystoscopy. Of the patients, 3\% progressed to muscle invasive disease at 2 years. This is the first study to confirm the previously published results (23).

A new paradigm for preoperative recurrence prophylaxis by one single intravesical electro-osmotic MMC instillation immediately before TURBT was recently presented (25). In a prospective study, 352 evaluable patients with urothelial NMIBC were randomized to three groups: TURBT alone ( $n=116$ ), TURBT followed by one single passive diffusion MMC $40 \mathrm{mg} / 50 \mathrm{ml}$ water instillation ( $n=119)$, or intravesical electro-osmotic MMC $40 \mathrm{mg} / 100 \mathrm{ml}$ water (electric current $20 \mathrm{~mA}$ for 30 minutes) before TURBT ( $n=117)$. All patients were followed-up, but patients with intermediate and high-risk disease also received long-term intravesical therapy according to standard protocols. Median follow-up was 84.7 months. The general trend of fewer recurrences was apparent in the group that received intravesical electro-osmotic MMC instillation 
TABLE II - Comparison of the outcomes of the study endpoints from the randomized trial comparing intravesical BCG alone and sequential BCG/electro-osmotic MMC in pT1 bladder cancer (23)

\begin{tabular}{|c|c|c|c|}
\hline & BCG alone & Sequential BCG/MMC & $\mathbf{p}$ \\
\hline - Patients (\%) & $61 / 105(58)$ & 45/107 (42) & 0.001 \\
\hline \multicolumn{4}{|l|}{ Progression to muscle invasive disease } \\
\hline - Patients (\%) & 23/105 (22) & 10/107 (9) & 0.005 \\
\hline \multicolumn{4}{|l|}{ Mortality } \\
\hline - Death from any cause (\%) & $34 / 105(32)$ & 23/107 (21) & 0.045 \\
\hline - Death from bladder cancer (\%) & 17/105 (16) & $6 / 107(6)$ & 0.010 \\
\hline
\end{tabular}

TABLE III - Comparison of the outcomes of the study endpoints from the randomized trial (median follow-up 85.4 months) comparing TUR alone, TUR andone single immediate postoperative intravesical passive diffusion MMC instillation, and one single immediate pre-TUR intravesical electro-osmotic MMC instillation (25)

\begin{tabular}{lccc}
\hline & TUR alone & $\begin{array}{c}\text { TUR andone immediate intravesical } \\
\text { passive diffusion MMC instillation }\end{array}$ & $\begin{array}{c}\text { One immediate pre-TUR intravesical } \\
\text { electro-osmotic MMC instillation }\end{array}$ \\
\hline $\begin{array}{l}\text { Number of patients } \\
\text { with recurrence }\end{array}$ & $74 / 116(64 \%)$ & $70 / 119(59 \%)$ & $44 / 117(38 \%)$ \\
$\begin{array}{l}\text { Median disease-free } \\
\text { interval months, (range) }\end{array}$ & $13(3-184)$ & $16(4-174)$ & $57(6-182)$ \\
\hline
\end{tabular}

before the TURBT (Tab. III). The overall median time to first recurrence was 57 months in pre-TURBT electro-osmotic MMC group compared with 13 months in TURBT/alone group and 16 months in post-TURBT passive diffusion MMC group. For electro-osmotic MMC pre-TURBT, 99\% of patients completed treatment. Intravesical passive diffusion MMC post-TURBT was not administered in $21 \%$ of patients, was prematurely stopped in $24 \%$, and was associated with persistent lower urinary tract symptoms exceeding those recorded after electro-osmootic MMC pre-TURBT and TURBT alone. Although this study queries the value of post-operative passive diffusion MMC instillations and may be a milestone for future therapeutic concepts, confirmation by other investigators is needed.

\section{Discussion}

There is a growing interest in methods to improve the delivery of intravesical chemotherapeutic agents to enhance efficacy. One example is intravesical electro-osmosis, which involves the application of electrical current across a biological barrier to accelerate the movement of drugs across the urothelium (17). Elecro-osomosis has been used to deliver a variety of intravesical drugs across the urothelium, including MMC for bladder cancer. In vitro $(14,17)$ and in vivo studies (19) demonstrated that electro-osmosis of MMC is more effective in delivering MMC into the urothelium, lamina propria, and superficial muscle layers of the bladder wall than is passive transport, and there is also less variability in the respective administration rates. Higher $\mathrm{MMC}$ concentrations may have a clinical impact, as intravesical electro-osmotic MMC provides a superior response rate in patients with high-risk superficial bladder cancer compared with passive diffusion MMC transport (19). Further data suggested that intravesical electro-osmotic MMC was at least equivalent to BCG in high-risk bladder tumors (19).

New strategies such as intravesical BCG combined with electro-osmotic MMC (23) as well as intravesical pre-operative electro-osmotic MMC (25) provided promising preliminary results in terms of higher remission rates and longer remission times.

Despite the level 1 evidence of efficacy, the schedule of sequential BCG/electro-osmotic MMC has not become the standard of care for high-risk NMIBC. Bachir et al (7) used statistical modeling to integrate costs and treatment efficacy rates to compare intravesical BCG alone to sequential BCG as well as electro-osmotic MMC. Examining drug costs, physician fees, and hospital costs, the authors estimated the impact of therapy on costs spanning 5 and 10 years, and found a significant financial benefit for combination therapy. The outcomes (23), cost-effectiveness analysis (7), and positive experiences reported by others (24) suggest that intravesical sequential BCG and MMC therapy can be potentially integrated into hospital and health system as a standard of care.

In conclusion, device-assisted intravesical chemotherapy may be a useful ancillary procedure in the treatment of 
patients with NMIBC. Its evaluation must be planned with respect to the technical functioning of equipment and their use for a clear purpose. This will help to avoid the financial and human costs associated with incorrect therapies, which can include poor patient care and unnecessary complications.

\section{Disclosures}

Financial support: The authors received no financial support for the research.

Conflict of interest: The authors declare that there are no conflicts of interest.

\section{References}

1. Ferlay J, Soerjomataram I, Ervik M, et al. GLOBOCAN 2012 v1.0, Cancer Incidence and Mortality Worldwide: IARC Cancer Base No. 11. Lyon, France: International Agency for Research on Cancer; 2013. Available at: http://globocan.iarc.fr. Accessed December 12, 2015.

2. Hall MC, Chang SS, Dalbagni G, Pruthi RS, Seigne JD, Skinner EC, Wolf JS Jr, Schellhammer PF. Guideline for the management of nonmuscle invasive bladder cancer (stages Ta, T1, and Tis): 2007 update. J. Urol. 2007;178:(6):2314-30.

3. Tolley DA, Parmar MK, Grigor KM, Lallemand G. The effect of intravesicalmitomycin $\mathrm{C}$ on recurrence of newly diagnosed superficial bladder cancer: a further report with 7 years of follow up. J Urol. 1996;155(4):1233-1238.

4. Botteman MF, Pashos CL, Redaelli A, Laskin B, Hauser R. The health economics of bladder cancer: a comprehensive review of the published literature. Pharmacoeconomics. 2003;21(18): 1315-1330.

5. Au JL, Badalament RA, Wientjes MG, et al. International Mitomycin C Consortium. Methods to improve efficacy of intravesicalmitomycin C: results of a randomized phase III trial. J Natl Cancer Inst. 2001;93(8):597-604.

6. Di Stasi SM, Riedl C. Updates in intravesical electromotive drug administration of mitomycin-C for non-muscle invasive bladder cancer. World J Urol. 2009;27(3):325-330.

7. Bachir BG, Dragomir A, Aprikian AG, et al. Contemporary cost-effectiveness analysis comparing sequential bacillus Calmette-Guerin and electromotive mitomycin versus bacillus Calmette-Guerin alone for patients with high-risk non-muscleinvasive bladder cancer. Cancer. 2014;120(16):2424-2431.

8. Bellantone NH, Rim S, Francoeur ML, Rosadi B. Enhanced percutaneous absorption via iontophoresis. Evaluation of an in vitro system and transport of model compounds. Int J Pharm. 1986;30:63-72.

9. Banga AK, Chien YW. Iontophoretic delivery of drugs: fundamentals, developments and biomedical applications. J Control Release. 1998;7(1):1-24.

10. Gangarosa LP, Park NH, Wiggins CA, Hill JM. Increased penetration of nonelectrolytes into mouse skin during iontophoretic water transport (iontohydrokinesis). J Pharmacol ExpTher. 1980;212(3):377-381.
11. Glass JM, Stephen RL, Jacobson SC. The quantity and distribution of radiolabeled dexamethasone delivered to tissue by iontophoresis. Int J Dermatol. 1980;19(9):519-525.

12. Vásquez JL, Gehl J, Hermann GG. Electroporation enhances mitomycin C cytotoxicity on T24 bladder cancer cell line: a potential improvement of intravesical chemotherapy in bladder cancer. Bioelectrochemistry. 2012;88:127-133.

13. Wientjes MG, Badalament RA, Au JL-S. Use of pharmacologic data and computer simulations to design an efficacy trial of intravesicalmitomycin C therapy for superficial bladder cancer. Cancer Chemother. Pharmacol. 1993;32(4)255-262.

14. Di Stasi SM, Giannantoni A, Massoud R, et al. Electromotive versus passive diffusion of mitomycin $C$ into human bladder wall: concentration-depth profiles studies. Cancer Res. 1999;59(19):4912-4918.

15. Nightingale ER Jr. Phenomenological theory of ion solvation. Effective radii of hydrated ions. J Phys Chem. 1959;63(9): 1381-1387.

16. Di Stasi SM, Giannantoni A, Massoud R, Cortese C, Vespasiani $G$, Micali F. Electromotive administration of oxybutynin into the human bladder wall. J Urol. 1997;158(1):228-233.

17. Di Stasi SM, Vespasiani G, Giannantoni A, Massoud R, Dolci S, Micali F. Electromotive delivery of mitomycin C into human bladder wall. Cancer Res. 1997;57(5):875-880.

18. Schmittgen TD, Wientjes MG, Badalament RA, Au JL-S. Pharmacodynamics of mitomycin $C$ in cultured human bladder tumors. Cancer Res. 1991;51(15):3849-3856.

19. Di Stasi SM, Giannantoni A, Stephen RL, et al. Intravesical electromotive mitomycin $C$ versus passive transport mitomycin $C$ for high risk superficial bladder cancer: a prospective randomized study. J Urol. 2003;170(3):777-782.

20. Brausi M, Campo B, Pizzocaro G, et al. Intravesical electromotive administration of drugs for treatment of superficial bladder cancer: a comparative Phase II study. Urology. 1998;51(3): 506-509.

21. Riedl CR, Knoll M, Plas E, Pflüger H. Intravesical electromotive drug administration technique: preliminary results and side effects. J Urol. 1998;159(6):1851-1856.

22. Colombo R, Brausi M, Da Pozzo L, et al. Thermo-chemotherapy and electromotive drug administration of mitomycin $\mathrm{C}$ in superficial bladder cancer eradication. A pilot study on marker lesion. Eur Urol. 2001;39(1):95-100.

23. Di Stasi SM, Giannantoni A, Giurioli A, et al. Sequential BCG and electromotive mitomycin versus BCG alone for high-risk superficial bladder cancer: a randomised controlled trial. Lancet Oncol. 2006;7(1):43-51.

24. Gan C, Amery S, Chatterton K, Khan MS, Thomas K, O'Brien T. Sequential bacillus Calmette-Guérin/electromotive drug administration of mitomycin $\mathrm{C}$ as the standard intravesical regimen in high risk nonmuscle invasive bladder cancer: 2-year outcomes. J Urol. 2016;195(6):1697-1703.

25. Di Stasi SM, Valenti M, Verri C, et al. Electromotive instillation of mitomycin immediately before transurethral resection for patients with primary urothelial non-muscle invasive bladder cancer: a randomised controlled trial. Lancet Oncol. 2011;12(9):871-879. 